\title{
La temporalité dans l'image: le cas de la métamorphose
}

\author{
FRANÇOISE FRONTISI-DUCROUX \\ Paris, Collège de France
}

\begin{abstract}
RESUMO: Estudando-se os procedimentos utilizados pelos pintores de vasos gregos para representar a metamorfose, verifica-se que os artistas optaram, freqüentemente, por recorrer a figuras híbridas, que se pode interpretar seja como em início de transformação, seja como uma alusão metonímica à metamorfose completada. Ao fazer-se um confronto com as narrativas correspondentes, percebe-se que os textos gregos também não descrevem o curso da metamorfose; contentam-se em mencioná-la rapidamente, sugerindo-Ihe o caráter imperceptível. 0 problema não é, portanto, unicamente figurativo: trata-se de uma impossibilidade mental e lingüística de pensar a duração e o movimento. As descrições minuciosas de Ovídio, que captam a transformação, alongando a duração, constituem, pois, uma notável inovação.
\end{abstract}

PALAVRAS-CHAVE: duração; híbrido; imagem; metamorfose; movimento; tempo.

La question est d'étudier l'expression de la temporalité dans les représentations figurées grecques de la métamorphose.

Bien qu'il s'agisse de confronter des images grecques, essentiellement des peintures de vases attiques à des textes en langue grecque, je commencerai cependant par deux passages d'Ovide: Mét III, 194-197 et II 476ss, où sont décrites les métamorphoses d'Actéon et de Callistô. Le premier décrit les modifications qu'impose Diane à Actéon en une suite d'actions au présent: elle applique des cornes sur sa tête, elle allonge son cou, elle épointe ses oreilles, change ses mains en pieds. Dans le second Ovide utilise le verbe coeperunt, inchoatif au parfait, suivi d'une série d'infinitifs, horrescere, curvari, crescere, fungi, fieri, verbes marquant, pour la plupart, le mouvement ou le devenir. Commencement et changement, c'est bien ainsi que nous concevons la métamorphose (surtout depuis que le cinéma s'en est emparé): une transformation progressive que le lecteur, ou l'auditeur, est invité à visualiser dans son déroulement temporel, en une succession d'étapes.

Face à cette représentation qui est nous est familière, voyons les images grecques.

Callistô n'est représentée qu'en céramique italiote du IV ème siècle; mais Actéon est présent sur la céramique attique.

Sa métamorphose, lorsqu'elle est indiquée (car elle ne l'est pas au début du Vème siècle: cf lécythes AFN, LIMC Aktaion 2 à 5) l'est généralement par la présence d'oreilles 
pointues et de cornes de cerfs sur la tête d'Actéon: cratères du milieu Vème siècle, LIMC Aktaion 81 et $83 a$.

C'est-à-dire par des "parties animales”, que l'on peut interpréter comme une amorce du processus, soit une marque de temporalité, la métamorphose débutant par les extrémités supérieures. Mais comme Actéon est (déjà) en proie à l'attaque de ses chiens, on peut comprendre ces indices comme une forme de synecdoque figurative (la partie pour le tout), imposée par un besoin de lisibilité: comment différencier un Actéon déjà totalement cerf d'un authentique cerf attaqué par une meute? sans doute n'est-il pas nécessaire de choisir entre les deux interprétations. C'est l'un et l'autre à la fois

La métamorphose est donc représentée par hybridation, en juxtaposant in praesentia, en une figure, deux états successifs, et ce dans un contexte narratif mettant en scène les événements qui se déroulent après la fin du processus de métamorphose (attaque des chiens)

Une étape antérieure en céramique attique présente un Actéon portant sur les épaules une peau de cervidé (biche) dont la tête coiffe celle d'Actéon - amphore FR Hambourg, de 490, LIMC Aktaion 27- procédure ambiguë qui peut dénoter aussi la tenue du chasseur et/ ou un trophée de chasse, et qui donne peut-être à voir l'illusion des chiens plutôt que la métamorphose effective (ce qui est un autre problème).

Une image exceptionnelle (fragment de l' Acropole du peintre de Pan) laisse entrevoir un Actéon dont le corps et les membres sont étroitement serrés dans une tunique de peau animale, solution originale qui superpose les deux états en donnant à voir simultanément (ou alternativement?) l'animalité et l'humanité du héros, l'avant et l'après. Il s'agit d'un cas exceptionnel qui probablemnt cherche à représenter aussi l'alternative entre métamorphose effective et folie hallucinatoire des chiens.

Au IVème siècle, dans la peinture italiote (ainsi que dans la statuaire puis dans la peinture pompéienne) ce type de représentation par hybridation, réduite aux extrémité supérieures: oreilles et cornes sera canonique: LIMC Aktaion, 38, 4548 etc.

Avec notre deuxième exemple, celui d'Io, nous observons les mêmes procédés: LIMC, Io 34 (cratère), 35 (oenochoé), 39 (skyphos).

L'hybridation minimale (femme munie de petites cornes) peut suggérer l'amorce de son animalisation, surtout si la figure est isolée.

Cette solution n'est cependant pas la plus courante dans la peinture attique. Souvent une vache est là dans son intégrité. D'autres composantes de l'image, la présence d'Hermès, du gardien Argos, voire celle de Zeus caressant la tête de son amante bovine, c'est-àdire le contexte, permettent alors l'identification.

En revanche une oenochoé lucanienne montre Zeus à côté d'une génisse à tête féminine: pouvant indiquer, si on l'interprète sur le plan temporel, la métamorphose en voie d'achèvement, mais aussi mettent en scène une formule inverse d'hybridation pour la vache Io.

Un dernier exemple retenu est celui des divinités marines.

Soit la série d'images centrées sur la lutte de Thétis et Pélée. Le héros enserre le corps de la déesse par une prise circulaire. Le couple est souvent entouré de Néreides, 
soeurs de Thétis, qui s'enfuient, paniquées. Des figures animales interviennent aussi, plus ou moins nombreuses: serpents se tortillant, panthère ou lion attaquant, agrippé sur le dos de Pélée et le mordant à l'épaule. Parfois Thétis brandit un serpent ou un poisson. Ou encore c'est une de ses soeurs qui tient le lion dirigé vers Pélée. Ailleurs des flammes jaillissent des épaules de Thétis Cf. LIMC, Peleus 87, 110, 112, 114, 160, 163, 175 etc Cet élément permet d'interpréter à coup sûr les figures animales qui assistent la déesse dans sa lutte comme les diverses formes qu'elle prend pour tenter d'échapper à Pélée. C'est que la métamorphose est, dans son cas, cyclique. Thétis endosse une série de formes avant de s'avouer vaincue - par l'étau circulaire des bras de Thésée-, et de se fixer dans l'anthropomorphisme, forme humaine féminine que montre toujours l'image, celle que Pélée pourra épouser.

Pour exprimer cette succession de métamorphoses, les peintres utilisent la juxtaposition. Ils accumulent dans l'espace iconique plusieurs figures qui correspondent chacune à un temps de la série. La présentation simultanée est la traduction spatiale de la succession chronologique. Mais si chargées soient-elle de temporalité, ces images n'en montrent cependant que des instants. Elles exposent les étapes d'un parcours de formes, sans s'arrêter sur le passage de l'une à l'autre. Une image fait peut-être exception, en insérant, parmi les animaux qui attaquent Pélée, une créature mixte, mi-lion, mi poisson Lécythe Syracuse 33501. On peut voir en cette hybridité une tentative d'enregistrer le changement et d'en fixer le moment.

Un premier bilan fait apparaître que la peinture céramique attique utilise pour la figuration de la métamorphose soit la juxtaposition, montrant in praesentia des moments successifs, soit l'hybridation, qui n'est qu'une sous catégorie de la juxtaposition. Soit encore le contexte.

Ce qui correspond à deux des trois procédés répertoriés par Alison Sharrock ${ }^{1}$, qu'elle nomme "incomplete representation" et "suggestion".

Le troisième procédé, nommé "panel" = panneau; qui correspond à notre bande dessinée ou tapisserie de Bayeux n'est pas attesté dans l'iconographie grecque, pour la métamorphose.

La conséquence en est que la figuration de la métamorphose est plutôt sommaire. La transition n'y est représentée qu'en une seule étape: l'achèvement complet, ou le tout début, ou une étape intermédiaire.

Cette observation est valable également aussi pour le dossier le plus ancien, celui de Circé, souvent utilisé dans les analyses de la narrativitée ${ }^{2}$ comme cas exemplaire de représentation synoptique du récit (évocation simultanée de plusieurs épisodes), question qui est différente.

Ainsi un lécythe (Tarente, Mus. Naz. 9125, LIMC "Kirke"15) met en scène Circé entourée de trois créatures identiques, à tête porcine, à pattes antérieures de porc, dont le corps anthropomorphe s'enrichit d'une queue tire-bouchonnée. S'il s'agit d'une phase de la métamorphose en cours, on voit que les victimes en sont toutes au même stade ${ }^{3}$. Comme dans le cas de la vache Io, la scène représente le résultat et ce sont les autres composantes de l'image qui permettent l'identification du sujet 
Mais sur la majorité des images des espèces diverses se côtoient et les victimes de la magicienne sont pourvues de têtes de cochon ou sang̉lier, cheval, âne, bélier, lion, panthère, chien et même cygne Par ex. Lécythe Tarente, Mus. Naz. 20324 (9877), LIMC "Kirke" 5.

Lorsque des images font apparaître des variations qui pourraient correspondre à des stades différents de la transformation en cours, un personnage à tête animale à côté d'un autre avec tête et sabots, l'image présente alors également un échantillonnage d'espèces différentes, ce qui indique un intérêt pour la variété formelle et pour l'hybridation en tant qu'occasion de recherche graphique. Ce qui est une constante de la représentation grecque de la métamorphose.

C'est également le cas pour l'hydrie étrusque de Toledo qui figure la transformation d'humains en dauphins (peut-être les pirates ravisseurs de Dionysos). Malgré ce que pourrait laisser croire un coup d'oeil trop rapide les six personnages qui plongent vers les flots ne correspondent pas à des stades différents de métamorphose. Cinq sont dauphins de la tête à la taille et le reste est humain. Déjà poissons, penserait-on, par la partie qui atteint les flots. Mais la créature de gauche interdit cette lecture, car, dans la même position, c'est la tête et le buste, ainsi que la main plongeant dans la vague, qui sont humains, alors que le reste est pisciforme. En revanche le peintre semble avoir voulu décomposer le mouvement de la plongée car les figures de droite ont les jambes inégalement dépliées, et leur allongement augmente progressivement de droite à gauche. Cette représentation du mouvement suppose un intérêt manifeste pour la temporalité et atteste un essai d'intégration, dans l'espace figuratif, d'un déroulement chronologique. Cependant, pour la métamorphose, plutôt que de montrer deux étapes différentes, ce peintre a choisi d'en représenter deux modalités: métamorphose procédant soit par le haut soit par le bas, chacune enregistrée au même stade. Ce qui donne en image deux formes d'hybridation possibles, entre l'homme et le dauphin (idem sur LIMC Kirke 19: un homme à tête et sabots de sanglier face à un sanglier debout sur ses pattes, mais à visage humain.)

C'est là, en réalité, ce qui, dans la métamorphose, intéresse les artistes, grecs autant qu'étrusques: le mélange des espèces, humaine et animale et ses diverses possibilités formelles. La métamorphose, qui permet d'explorer les frontières entre humanité et bestialité, est pour les peintres avant tout matière à expérimentation figurative.

Pas d'arrêt sur image, donc, ou fort peu, dans l'imagerie grecque, qui capterait et évoquerait une métamorphose dans son déroulement. Cela tient, pensera-t-on à la nature sychronique de l'image.

Pourtant si l' on se réfère aux textes grecs qui correspondent à ces images on constate qu'ils sont encore moins détaillés que les images.

Ainsi, pour Circé, dans l'Odyssée, (X 235-243 et 390-395) le récit est aussi expéditif que le pouvoir de la magicienne: elle accueille les voyageurs, leur verse à boire, "ajoutant ensuite au mélange un philtre qui devait leur faire oublier la patrie. Elle avança la coupe, qu'ils vidèrent; peu après, sur un coup de baguette, ils étaient bouclés dans l'étable. Des cochons ils avaient les groins, les grognements, les soies, tout enfin, sauf l'esprit qui resta esprit de mortel". "Les ayant frappés de sa baguette, elle les enferma", dit le texte, en utilisant l'aoriste. Puis l'imparfait echon, évoque l'état des marins devenus porcs. La métamor- 
phose même a été escamotée. L'opération inverse, exécutée sous la surveillance d'Ulysse, est à peine plus détaillée: "Elle les enduisit d'une drogue... leur poils tombèrent...ils redevinrent -egenonto - des hommes". Ici encore, l'aoriste est utilisé pour relater une opération ponctuelle et son résultat est présenté comme immédiat.

Et c'est là une constante des textes grecs. La métamorphose proprement dite n'y est que mentionnée et la sècheresse de l'énoncé en est un trait dominant. Rien, jamais, de semblable aux descriptions d' Ovide qui déploie complaisamment une transformation dans toutes ses étapes par des procédures verbales quasi cinétiques.

On s'en convaincra en lisant en $O d$. IV, 454-461 le récit par Ménélas de sa lutte contre le Vieux de la Mer, qu'il a guetté patiemment, dissimulé avec ses compagnons sous d'immondes peaux de phoques: "C'est alors qu'en hurlant nous bondîmes pour l'empoigner: mais le malin vieillard n' ayant point oublié ses tours, d'abord se transforma en lion de belle crinière, puis en dragon, puis en panthère, puis en porc. Nous cependant le tenions ferme, patiemment. Mais lorsque le vieillard eut épuisé ses sortilèges, il se tourna vers moi et me posa cette question..." Ce cycle de métamorphoses analogues à celles de Thétis, ne donne lieu, de la part du poète, qu'à une énumération des figures successivement endossées, et présentées par le verbe geneto.

Il en va de même, dans l' Hymne homérique à Dionysos (34ss). On peut constater une opposition entre la descriptions des prodiges suscités par Dionysos sur le navire des pirates: le vin qui coule, la vigne qui s'étend, les grappes pendantes, le lierre qui s'enroule sur le mât..., où les imparfaits duratifs alternent avec les aoristes, et l'instantanéité de la métamorphose du dieu "il devint -egeneto- un lion qui poussait de terribles rugissements; et de celle des pirates qui, affolés, sautèrent dans la mer et y devinrent des dauphins -delphines egenonto

La lecture des textes révèle ainsi que les écrivains grecs n'utilisent guère les ressources temporelles du langage et son aptitude à se déployer dans la durée, pour dérouler les étapes d'une transformation.

Notre dossier textuel comporte certes une lacune: nous ne connaissons le poème de Nicandre, modèle principal d'Ovide, dit-on, que par les résumés d'Antoninus Liberalis, qui se contentent d'énoncer l'événement. Antoninus Liberalis emploie généralement les verbes poiein, tithenai, et metaballein, "faire" et transformer", parfois metamorphoun ou (met)allattein, "métamorphoser" et "changer", toujours à l'äoriste, pour dire une transformation exécutée par un sujet, et fréquemment: egeneto, "il devint", pour la métamorphose subie. On pourrait donc attribuer à la convention du résumé le caractère purement ponctuel de ce mode d'expression, et espérer que l'ouvrage perdu de son prédécesseur était plus riche en détails évocateurs. Ce n'est pourtant pas du tout l'impression que donne un fragment de Nicandre relatant la métamorphose d'Hécube en chienne, texte assez pauvre qui énonce simplement "elle changea sa forme de vieille femme, se faisant semblable aux chiennes d'Hyrcanie" Ellaxato morphen greion, Hyrkanessin eeidomenèn skulakessin ( Nicandre, fr. 62, éd Gow-Scholfield,)en utilisant, ici encore l'aoriste.

L'aoriste, ou indéfini, semble bien une constante de l'expression grecque de la métamorphose.

En grec, nous le savons, la notion d'aspect désigne les différentes perspectives sous lesquelles apparait une action, que l'expression verbale peut envisager, soit dans son déve- 
loppement, par le duratif, qu'exprime le thème du présent, soit comme ramassée en un point, par le ponctuel, que prend en charge le thème de l' aoriste. Et l'opposition est constante entre duratif -présent et imparfait- et aoriste ponctuel. L'un donne à l'action une durée intéressante: l'imparfait marque un intérêt pour le développement de l'action décrite; tandis que l'autre fait négliger la durée: l'aoriste est ainsi le temps de la chronologie pure et du procès verbal. Le grammairien Jean Humbert, insiste sur caractère négatif de l'aoriste: "l'aoriste constate un fait dont la durée n'a pas d'intérêt aux yeux du sujet parlant", et il montre qu'une même action peut être décrite par l'aoriste ou par l'imparfait. La conception que les Stoiciens, (cités ici par J. Humbert), inventeurs du terme "aoriste", se font du temps nous fournit une grille permettant d'appréhender le problème. Selon Arius Didyme, Chrysippe définit le temps comme "intervalle du mouvement au sens où on l'appelle parfois mesure de la rapidité ou de la lenteur". Dans l'aoriste le poson temporel, la quantité de temps est indéfinie. L’indéfini annule la détermination.

Or nous venons de voir que les écrivains grecs ne choisissent jamais, comme le fait Ovide en latin, de dérouler les étapes d'une métamorphose en utilisant la valeur aspectuelle du présent et de l'imparfait, qui servent à indiquer qu'une action est envisagée dans son développement. Tout au contraire, l'aoriste qu'ils emploient régulièrement semble marquer un refus ou une impossibilité d'envisager, à propos de la métamorphose, la durée.

Le problème n'est ainsi ni purement iconographique ni, non plus, "psychologique". Nous dirons qu'il est d'ordre anthropologique. Ce qui doit entrer en ligne de compte, c'est la conception de la métamorphose, qui n'est pas un fait, mais une donnée culturelle et construite.

L'examen de quelques autres textes permettar de préciser la question. Chez Homère, les métamorphoses des dieux eux-mêmes se caractérisent par leur rapidité et leur instantanéité. Ainsi Athéna en Odyssée XXII, 205 et 239: elle se se matérialise "avec l'allure et la voix de Mentor", sans que personne en soit témoin puis disparait "sous la forme d'une hirondelle" sans que nul ne remarque rien. En Odyssée III, 372, sa métamorphose en oiseau est un moyen de quitter les mortels en même temps que sa forme humaine, celle de Mentor, et l'assistance s'émerveille et reconnaît la divinité. Dans les deux cas nul changement n'est décrit: un verbe pour dire l'envol, et une comparaison ou une épithète disant la ressemblance pour indiquer la forme déjà prise. Ces métamorphoses divines sont surtout des disparitions.

Le prodige d'Aulis raconté en Iliade, II, 306-320 est explicite. Quand le terrible serpent "appelé à la lumière par le dieu de l'Olympe" a dévoré les passereaux, "le dieu qui l'avait fait paraître le déroba à nos yeux: le fils de Cronos le Fourbe l'avait soudain changé en pierre. Nous restions là, immobiles, à admirer l'événement".

L'énoncé "Zeus pétrifie le serpent: "laan min etheken: il le fit devenir pierre" est explicatif du vers précédent: "le dieu le rendit invisible -ton men aizelon theken theos "(aoriste). La pétrification, variété de métamorphose, est présentée moins comme passage de l'animal au minéral, que comme un mode de disparition totale. Disparition qui répond à l'apparition initiale du serpent suscitée par le même Zeus.

Dans cet épisode, le prodige, apparition/ disparition du serpent, est un spectacle, qui fige de stupeur l'assemblée. Sous les yeux émerveillés des Grecs, Zeus escamote le monstre 
en le transformant en pierre. L'opération, quoique donnée à voir, échappe aux regards en échappant à la durée

Cette métamorphose s'inscrit dans le temps d'un battement de paupière. Elle n'est pensée que comme le remplacement d'un état par un autre, en un clin d'oeil, c'est-à-dire dans le temps de l'obstruction de la vision. Pour les Grecs tout se passe comme si la métamorphose était du non-visible. Parce que l'action divine est non-visble, de même que les dieux sont invisibles aux humains (telle Athéna chez Eumée) dont la vue trop faible. On comprend que l'aoriste soit mobilisé pour dire cette "annulation de l'intervalle du mouvement", "du poson temporel”, selon les Stoiciens. Il s'agit du temps indéfini des dieux.

Cette appréhension toute ponctuelle de la métamorphose, atemporelle parce qu'imperceptible, à la fois spectaculaire et in-visible, perdure bien au-delà des poèmes homériques.

Ainsi de la substitution qu'opère Artémis lors du sacrifice d'Iphigénie. La déesse enlève la jeune fille et dépose une biche à la place. Il s'agit d'un échange et non d'un changement: c'est ce qu'indique le préfixe anti utilisé tant par Euripide (Iph. Taur. 28: antidousa) que par Antoninus Liberalis, XXVII. Et ce dernier précise que la substitution s'est opérée au moment où les Grecs détournaient leurs regards de l'horrible sacrifice; ou baissaient la tête, selon Euripide.

Est-ce Ovide qui a inventé le ralenti? c'est vraisemblable étant donné l'ampleur de son projet. Ses premier mots = in nova (même si on s'aperçoit que l'épithète s'applique à corpora en rejet au vers suivant) marquent la volonté d'une rénovation complète du sujet. Certes en latin il n'y a pas d'aoriste, mais le parfait peut en tenir lieu et Ovide ne manque pas de varier considérablement les approches. Le plus souvent cependant il étire l'événement, jusqu'alors imperceptible, en tout cas en langue grecque, en l'inscrivant dans la durée du temps humain, du regard humain, et donne en spectacle ce qui avant échappait, sauf peut-être à la vision du poète, de l'aède, qui mentionnait et expliquait la métamorphose sans pouvoir la donner à voir, à son auditeur.

Je ne sais s'il s'agit là d'une autre conception du divin. Ovide met en scène dans ses Métamorphoses des dieux opérant à tour de bras. Il est certain qu'il s'agit d'un grand projet esthétique ${ }^{4}$.

En conclusion je ferai quelques remarques d'ordre général:

Bien qu'il faille, dans l'étude des images, postuler une autonomie des traditions figuratives, le rapprochement images/ textes est particulièrement justifié dans le cas des images dites "mythologiques". Pas uniquement parce que nous ne pouvons les comprendre qu'en nous référant à des textes anciens. Mais surtout parce que pour les imagiers et leur public le contenu de ces images c'est du muthos, du discours. Il y a une nécessaire médiation verbale entre l'événement mythique et l'image conçue et produite par le peintre (nul n'a assisté de visu à l'une de ces métamorphoses). Ce discours pouvait être oral ou écrit. Nous n'avons qu'une petite partie du second, le premier nous est totalement inaccessible.

Lorsqu'il s'agit d'un récit mythique et de ses versions iconographiques, il nous est impossible de savoir quel genre de récit l'artiste avait en tête: un texte littéraire, poétique, fixé ou oral, ou un récit minimal, dont l'ordre temporel (celui de la disposition des séquences), transcrirait de façon linéaire l'ordre chronologique des événements. Ce degré zéro du récit est rare, en dehors des résumés des mythographes. 
L'art du récit, oral et plus encore écrit, consiste souvent à créer des discordances entre les deux temps. Distorsions que G. Genette nomme anachronies, telles les anticipations-ou prolepses- et les retrospections ou analepses ${ }^{5}$.

Le problème se pose par exemple pour l'épisode de Circé, exemple privilégié, en raison de son caractère synoptique, des analyses de la temporalité dans l'image ${ }^{6}$, car le récit correspondant auquel nous avons accès, celui de l'Odyssée - mais dans quel état du texte?- comporte d'importantes distorsions temporelles, et répétitions, dues à l'intervention d'Hermès qui explique à Ulysse ce qui est arrivé à ses compagnons et lui annonce ce qui va se passer.

Mais le problème que nous envisagions ici, celui des figurations de la métamorphose ne concerne pas l'ordre du récit (les récits où sont inclus des métamorphoses peuvent y ressortir). La représentation de la temporalité propre au procès de métamorphose relève du problème de la durée, qui est une notion culturelle. En ce cas le recours au texte est le seul moyen pour nous d'atteindre aux structures mentales et imaginaires des producteurs d'images et des destinataires de ces images. Nous avons vu que les structures linguistiques et grammaticales peuvent fournir des solutions. C'est l'expression linguistique du temps qui révèle la conception du temps, même figuré en image.

\section{Notes}

1 - "Representing metamorphosis" in Elsner ed. Art and Text in Roman culture. Cambridge, 1996, 103-130.

2 - en particulier par A. Snodgrass, "La naissance du récit dans l'art grec", Images et société en Grèce ancienne, Cahiers d'Archéologie romande n 36, Lausanne, 1987, 11-18.

3 - La métamorphose complète est attestée sur un vase très fragmentaire de Brygos qui montre Ulysse à côté d'un sanglier, d'une panthère et d'un autre fauve: Athènes Acr. 293 (B 66) cf. O. Touchefeu-Meynier, Thèmes odysséens dans l'art antique, Paris 1968, p 91 note 15.

4 - Ce que Galinsky nomme "visual over explicitness": Ovid's Metamorphoses, Oxford, 1975.

5 - Figures III, Paris, 1972.

6 - Cf. supra note 2.

\section{Bibliographie}

GALINSKY, G. K. Ovid's Metamorphoses. Oxford: 1975.

GENETTE, G. Figures III. Paris: 1972.

SHARROCK, A. "Representing metamorphosis". In: ELSNER (ed). Art and text in Roman culture.Cambridge: 1996, 103-130.

SNODGRASS, A. Homer and the artists. Cambridge: 1999. 
FRONTISI-DUCROUX, Françoise. La temporalité dans l'image: le cas de la métamorphose. Classica, São Paulo, 13/14, p.241-249, 2000/2001

RÉSUMÉ: En étudiant les procédures utilisées par les peintres de vases grecs pour représenter la métamorphose on constate que les artistes ont le plus souvent choisi de recourir à des figures hybrides, que l'on peut interpréter soit comme en début de transformation, soit comme une allusion métonymique à la métamorphose achevée. Or si l'on se réfère aux récits correspondants on découvre que les textes grecs ne décrivent pas davantage le cours de la métamorphose, mais se contentent de l'énoncer brièvement en suggérant son caractère imperceptible. Le problème n'est donc pas uniquement figuratif: il s'agit d'une impossiblité mentale et linguistique de penser la durée et le mouvement. Les descriptions minutieuses d'Ovide qui captent la transformation en étirant la durée constituent donc une innovation remarquable.

MOTS-CLÉS: durée; hybride; image; métamorphose; mouvement; temps. 